\title{
EFFECTS OF FACILITY AND SERVICE QUALITY ON EXPERIENCE VALUE, SATISFACTION, AND BEHAVIORAL INTENTIONS IN AN URBAN THEME PARK
}

\author{
Chung-Tai $\mathrm{Wu}^{1}$, Ting-Wen Wang ${ }^{2}$ \\ ${ }^{1}$ Department of Tourism \& Leisure, Lee-Ming Institute of Technology, Taiwan \\ ${ }^{2}$ Department of Food \& Beverage, Lee-Ming Institute of Technology, Taiwan
}

DOI: 10.46609/IJSSER.2020.v05i05.014 URL:https://doi.org/10.46609/IJSSER.2020.v05i05.014

\begin{abstract}
The study uses multiple regressions to explore the relationships among five constructs: "theme park facilities", "service quality", "experience value", "satisfaction", and "behavioral intention". The results showed facilities to affect experience value more than the effect from service quality. A chain effect from experience value to satisfaction and behavioral intentions were identified. Facilities also affect satisfaction and behavioral intentions much more than the effect from service quality. Demographics differences were also explored though one-way ANOVA, which paved the way for recommendations.
\end{abstract}

Keywords: Theme park facilities, Service quality, Experience value, Satisfaction, Behavioral intentions

\section{INTRODUCTION}

Theme park is an attractive option for leisure activities in an urban setting due to limited open space (Buckley, 2000; Dzeng \& Lee, 2007). Economic forces, employees, and changing demographic forces have been perceived to have the greatest impact on the operation of the theme park industry (Samuels, 1996; Milman, 2001). Consumers may seek interactive adventure, fantasy and mystery, movies and television shows, and science fiction/futuristic themes, as well as the quality of service provided. Bitner (1992) is one of the first pioneers who explored the impact of physical surroundings on customers. Later, Hightower et al. (2002) explored the relationship between physical environment (servicescapes) and key service constructs (positive effect, enduring involvement, service quality, waiting time, and value) 


\section{International Journal of Social Science and Economic Research}

ISSN: $2455-8834$

Volume: 05, Issue: 05 "May 2020"

consumers' behavioral intentions.

Other than physical surroundings, a Scottish study found that fun rides, little waiting, and good climate or environment were the most important choice criteria for amusement park visitor behavior (Moutinho, 1988). McClung (1991) attributes distance, cost, crowds, preference, climate lodging, and children to the selection of theme park visitation. Relative importance of factors tourists rely on in evaluation of theme parks that satisfy their motives may be analyzed through importance-performance technique (Haahti \& Yavas, 2004). There is also a distinct gender difference in the perception of leisure activities (de Fontenelle \& Zinkhan, 1993). Hence, the study seeks to explore dimensions affecting customers' behavioral intensions toward theme parks, as well as demographic differences on each dimension.

\section{LITERATURE REVIEW}

A study of experiential quality, experiential value, experiential satisfaction, theme park image, and revisit intention revealed four primary dimensions and eleven sub-dimensions of experiential quality perceived by theme park visitors (Wu et al., 2018). In addition physical environment quality was also identified as the most primary dimension of experiential quality perceived by them park visitors.

Kim et al. (2003) identified four factors (family togetherness, appreciating natural resources, escape from routine, and adventure) that push people to theme parks and three pull factors (key tourist resources, information and convenience of facilities, and accessibility/transportation). Millman (2009) found identified seven major factors influencing guest experience at theme parks: (1) entertainment variety and quality, (2) courtesy, cleanliness, safety, and security, (3) food variety and value for money, (4) theme quality and design, (5) availability and variety of family-oriented activities, (6) quality and variety of rides and attractions, and (7) pricing and value for money. A study of "facility-driven" leisure services found the more theme park visitors perceive physical surroundings as novel, the higher of perceived values (utilitarian and hedonic) and the stronger their intention to revisit (Chang et al., 2014).

There is a unique relationship between the service facility placements and the amount of predicted traffic flows to theme parks (Hyun et al., 2016). Zhang et al. (2017a) studied how attraction and spatial layout attributes affect them park visitor movement where it was found that visitor movement is influenced by attraction attributes such as attraction type, experience value, facility capacity, floor area, and indoor feature, while spatial layout attributes include: distance between attractions, path network, entrance location, and attraction distribution. Theme park attendance affects visitors' experience, satisfaction, and their behavioral intentions directly and/or indirectly (Zhang et al., 2017b). 


\section{International Journal of Social Science and Economic Research}

ISSN: $2455-8834$

Volume: 05, Issue: 05 "May 2020"

In a case study of cultural them park, it was found that theme park brand satisfaction is related to brand loyalty, while the quality of facilities and attractions has no significant correlation with perceived value and brand satisfaction (Cheng et al., 2016). Also, no significant correlation was found between service quality and perceived value. Nonetheless, there is a linkage between human resource practices and the delivery of guest services in theme park operation in terms of the significance of service quality (Mayer, 2002). Service design offers effective methods for analyzing and developing complex service experiences (Trischler \& Zehrer, 2012). In addition, the multistep approach delivers comprehensive insights into customer experiences and identifies critical incidents that take place during the service experience. Planning service facilities applies to operation, location, atmospheric and image, consumer use, and contact personnel as multidimensional nature of service facilities (Turley \& Fugate, 1992).

\section{METHODOLOGY}

The literature review provided the bases of constructing the questionnaire. Five constructs were established: "theme park facilities", "service quality", "experience value", "satisfaction", and "behavioral intentions". The "facilities" dimension includes six items: (1) theme park route is smooth; (2) sign direction is clear; (3) facilities are fun; (4) facilities are diverse; (5) novelty on most of the facilities; and (6) facilities are safe. The "service quality" construct includes ten items: (1) good hygiene/sanitation; (2) clean and efficient parenting area; (3) provides trustworthy service in a timely fashion; (4) perceived comfort; (5) extra attention on toddlers; (6) easy accessibility to service providers; (7) convenient business hour; (8) provides individual service; (9) does not overlook customers' needs; and (10) provider's attitude does not vary with different customers. The "experience value" construct includes eight items: (1) collocated facilities within the surrounding; (2) gives my family fun; (3) clear image for people of all ages; (4) facilities and programs fit families with children; (5) family relationship enhanced by the theme park experience; (6) facilities fits the theme of this park; (7) dining options are diverse; and (8) theme park atmosphere is joyous. Three items that measure "satisfaction" are as follows: (1) satisfied with the overall services; (2) theme park entry price is reasonable; (3) satisfied with the offered facilities overall. At last, three conventional items that measure "behavioral intentions" are: (1) revisit intention, (2) recommendation to family and/or friends, and (3) top choice of my future visit among many options.

The answered questionnaires were rated on a five-point Likert-type scale, ranging from 1 for strongly disagree to 5 for strongly agree. The data were then analyzed through a popular statistical program, SPSS 20 for Windows. After formulation of the questionnaire, 200 copies of the questionnaires were distributed to visitors of the selected theme park in Taipei City. The survey took place from February $20^{\text {th }}$ to December $15^{\text {th }}$ of 2019 . From 200 returns, 180 
answered questionnaires were deemed valid. The demographic profile of the survey is illustrated in Table 1 , where $26 \%(n=47)$ of the survey were answered by male, and $74 \%(n=133)$ were answered by female. Other demographic characteristics of the theme park visitors are: marital status, age, education, occupation, number of accompany in this particular visit, number of children under 12 years-old in the visiting party, and the visitation number within a year, where each sample number and respective percentage are shown.

\section{RESULTS AND DISCUSSION}

The one-way ANOVA results are shown in Table 2 (gender), Table 3 (marital status), Table 4 (age group), Table 5 (post-hoc of age groups), Table 6 (education level), Table 7 (occupation), Table 8 (accompany number), Table 9 (number of accompanied children), Table 10 (post-hoc of accompanied children), and Table 11 (times of visit in a year). Results showed insignificance by variance of either gender or marital status. However, by the age group, visitors aged between 26 and 35 years-old are more dissatisfied with the theme park's "facilities" than those of other age groups. The rational of this finding is that the age group of 26-35 are people entering the workforce, may have lesser financial resources, and may demand more return from their spending (on park fees). Hence, they rated "facilities" lower than visitors of other age groups. Conversely, visitors aged between 36 and 45 years-old have much higher "behavioral intentions" toward revisit of the theme park than visitors of other age groups. The rational of this finding would work along side the previous trend that this age group (36-45 years-olds) may have more financial security and spending power, thereby more likely to pay a return visit. In addition, this particular age group consisted of people more likely to have young children to bring to the theme park than visitors of other age groups. The variance significance by age group is validated by post hoc analysis where least-significant-difference (LSD) displayed satisfaction on both "facilities" and "behavioral intentions" but the more rigorous method (Scheffe comparison) is only satisfied for "behavioral intentions". 
Table 1: Demographic characteristics of the respondents $(n=180)$

\begin{tabular}{llcc}
\hline Demographics & & Number & Percentage \\
\hline Gender & Male & 47 & 26 \\
Marital Status & Female & 133 & 74 \\
Age & Single / Divorced / Widowed & 85 & 47 \\
& Married & 95 & 53 \\
& $18-25$ years old & 62 & 34 \\
Education & $26-35$ years old & 45 & 25 \\
& 36-45 years old & 34 & 19 \\
& 46 years old or older & 39 & 22 \\
Occupation & High school or less & 61 & 34 \\
& 4-year college & 111 & 62 \\
& Post graduate & 8 & 4 \\
Accompany (include self) & Self-employed & 13 & 7 \\
& Service industry & 45 & 25 \\
& Governmental & 6 & 3 \\
Accompanied child & Others & 116 & 65 \\
& 3 persons or less & 80 & 44 \\
& 4-6 persons & 82 & 46 \\
& 7 persons or more & 18 & 10 \\
& None & 77 & 43 \\
Visitation number & 1 child & 43 & 24 \\
(within a year) & 2 children & 44 & 24 \\
& 3 children or more & 16 & 9 \\
& 1 time & 115 & 64 \\
& 2 times & 43 & 24 \\
& 3 times or more & 22 & 12 \\
\hline & & &
\end{tabular}

Table 2: Visitors' response towards each construct (by gender) 
International Journal of Social Science and Economic Research

ISSN: $2455-8834$

Volume: 05, Issue: 05 "May 2020"

\begin{tabular}{llrlccc}
\hline Construct & & $N$ & Mean & S.D. & F-value & Sig. \\
\hline Facilities & Male & 47 & 3.8333 & 0.44910 & 0.833 & .363 \\
Service quality & Female & 133 & 3.7556 & 0.51882 & & \\
& Male & 47 & 3.7298 & 0.36708 & 0.538 & .464 \\
Experience value & Female & 133 & 3.6827 & 0.38188 & & \\
& Male & 47 & 4.1011 & 0.32307 & 0.035 & .851 \\
Satisfaction & Female & 133 & 4.0902 & 0.34589 & & \\
& Male & 47 & 3.8156 & 0.55084 & 3.056 & .082 \\
Behavioral intentions & Female & 133 & 3.7549 & 0.43767 & & \\
& Male & 47 & 3.9433 & 0.63823 & 1.378 & .242 \\
& Female & 133 & 3.8269 & 0.54528 & & \\
\hline
\end{tabular}

${ }^{*} p<.05 ;{ }^{* *} p<.01 ;{ }^{* * *} p<.001$

Table 3: Visitors' response towards each construct (by marital status)

\begin{tabular}{llccccc}
\hline Construct & & $N$ & Mean & S.D. & F-value & Sig. \\
\hline \multirow{2}{*}{ Facilities } & Single/Divorced/ Widowed & 85 & 3.8333 & 0.44910 & 2.087 & .127 \\
& Married & 95 & 3.7556 & 0.51882 & & \\
Service quality & Single/Divorced/ Widowed & 85 & 3.7907 & 0.54421 & 2.083 & .128 \\
& Married & 95 & 3.7456 & 0.45924 & & \\
Experience value & Single/Divorced/ Widowed & 85 & 3.8561 & 0.39287 & 2.350 & .098 \\
& Married & 95 & 3.7411 & 0.36217 & & \\
Satisfaction & Single/Divorced/ Widowed & 85 & 4.1250 & 0.37680 & 0.734 & .481 \\
& Married & 95 & 4.0553 & 0.30166 & & \\
Behavioral & Single/Divorced/ Widowed & 85 & 3.9309 & 0.55367 & 0.844 & .432 \\
intentions & Married & 95 & 3.8982 & 0.39522 & & \\
\hline
\end{tabular}

${ }^{*} p<.05 ;{ }^{* *} p<.01 ;{ }^{* * *} p<.001$

Table 4: Visitors' response towards each construct (by age group) 
International Journal of Social Science and Economic Research

ISSN: $2455-8834$

Volume: 05, Issue: 05 "May 2020"

\begin{tabular}{llllllc}
\hline Construct & & $N$ & Mean & S.D. & F-value & Sig. \\
\hline Facilities & 18-25 years old & 62 & 3.8360 & 0.56820 & 3.789 & $.011^{*}$ \\
& 26-35 years old & 45 & 3.5667 & 0.40107 & & \\
& 36-45 years old & 34 & 3.8922 & 0.45298 & & \\
& 46 years old or older & 39 & 3.8205 & 0.47968 & & \\
Service quality & 18-25 years old & 62 & 3.8435 & 0.39156 & 2.311 & .078 \\
& 26-35 years old & 45 & 3.7244 & 0.41350 & & \\
& 36-45 years old & 34 & 3.8941 & 0.32468 & & \\
Experience value & 46 years old or older & 39 & 3.7128 & 0.33260 & & \\
& 18-25 years old & 62 & 4.0988 & 0.35001 & 1.920 & .128 \\
& 26-35 years old & 45 & 4.0083 & 0.37914 & & \\
Satisfaction & 36-45 years old & 34 & 4.1912 & 0.32746 & & \\
& 46 years old or older & 39 & 4.0962 & 0.26198 & & \\
& 18-25 years old & 62 & 3.9677 & 0.58428 & 1.697 & \\
& 26-35 years old & 45 & 3.8370 & 0.41208 & & \\
& 36-45 years old & 34 & 4.0294 & 0.37933 & & \\
Behavioral & 46 years old or older & 39 & 3.8376 & 0.38897 & & \\
intentions & 18-25 years old & 62 & 3.7742 & 0.72873 & 4.515 & \\
& 26-35 years old & 45 & 3.8370 & 0.33801 & & \\
& 36-45 years old & 34 & 4.1569 & 0.44370 & & \\
\hline & 46 years old or older & 39 & 3.7607 & 0.52953 & & \\
\hline
\end{tabular}

${ }^{*} p<.05 ;{ }^{* *} p<.01 ;{ }^{* * *} p<.001$

Table 5: Post-hoc test by LSD and Scheffe for age group 


\begin{tabular}{|c|c|c|c|c|}
\hline Construct & $(\mathrm{I})>(\mathrm{J})$ & Difference & Sig. & Method \\
\hline \multirow[t]{6}{*}{ Facilities } & \multirow[t]{2}{*}{$(A)>(B)$} & \multirow[t]{2}{*}{0.26935} & $.006^{2+}$ & LSD \\
\hline & & & .052 & Scheffe \\
\hline & \multirow[t]{2}{*}{$(\mathrm{C})>(\mathrm{B})$} & \multirow{2}{*}{0.32549} & $.004^{* *}$ & LSD \\
\hline & & & $.039^{\circ}$ & Scheffe \\
\hline & \multirow[t]{2}{*}{ (D) $>$ (B) } & \multirow{2}{*}{0.25385} & $.019^{*}$ & LSD \\
\hline & & & .137 & Scheffe \\
\hline \multirow[t]{4}{*}{ Behavioral intentions } & $(\mathrm{C})>(\mathrm{A})$ & 0.38267 & $\begin{array}{l}.002^{* *} \\
018^{*}\end{array}$ & $\begin{array}{l}\text { LSD } \\
\text { Scheffe }\end{array}$ \\
\hline & \multirow{2}{*}{$(\mathrm{C})>(\mathrm{B})$} & \multirow{2}{*}{0.31983} & $.012^{*}$ & LSD \\
\hline & & & .098 & Scheffe \\
\hline & $(\mathrm{C})>(\mathrm{D})$ & 0.39618 & $\begin{array}{l}.003^{* *} \\
.029^{*}\end{array}$ & $\begin{array}{l}\text { LSD } \\
\text { Scheffe }\end{array}$ \\
\hline
\end{tabular}

The results (Tables 6, 7, and 8) displayed insignificance by the variance of education level, occupation, and accompanied number (total of adult/child) of the park visit. However, the variance of accompanied child number (Table 9) would show significance. Post hoc analysis (Table 10) showed that visitors with two children are much more satisfied with the theme park's "facilities", "service quality", and "experience value", as well as higher "behavioral intentions" toward a repeat visitation than those with other accompanied child numbers. The validation exhibited more significance toward the construct of "facilities" and "behavioral intentions" than the construct of "service quality" and "experience value. The rational of the finding is that people with three children or more may have a larger age disparity among their children; hence, making their family more difficult to enjoy the park's "facilities" and "service quality". On the other hand, visitors with two children may have a much smaller gap of age difference, allowing their family to enjoy the park's "facilities", "service quality", and "experience value" more easily together; hence, their "behavioral intentions" much higher than the other groups. Although visitors with no child or one child enjoyed the park's "facilities", their level of enjoyment is just not as high as those with two children. At last, Table 11 showed that the variance of park visitation frequency played no significance toward any of the constructs.

Table 6: Visitors' response towards each construct (by education) 
International Journal of Social Science and Economic Research

ISSN: 2455-8834

Volume: 05, Issue: 05 "May 2020"

\begin{tabular}{llrrrrc}
\hline Construct & & $N$ & Mean & S.D. & F-value & Sig. \\
\hline Facilities & High school or less & 61 & 3.8005 & 0.46926 & 0.435 & .648 \\
& 4-year college & 111 & 3.7733 & 0.51138 & & \\
& Post graduate & 8 & 3.6250 & 0.63465 & & \\
Service quality & High school or less & 61 & 3.7377 & 0.30885 & 3.018 & .051 \\
& 4-year college & 111 & 3.8063 & 0.39480 & & \\
& Post graduate & 8 & 4.0750 & 0.50920 & & \\
Experience value & High school or less & 61 & 4.0451 & 0.26124 & 1.764 & .174 \\
& 4-year college & 111 & 4.1284 & 0.36520 & & \\
& Post graduate & 8 & 3.9688 & 0.45193 & & \\
Satisfaction & High school or less & 61 & 3.8306 & 0.40653 & 1.730 & .180 \\
& 4-year college & 111 & 3.9580 & 0.50476 & & \\
& Post graduate & 8 & 4.0417 & 0.41547 & & \\
Behavioral & High school or less & 61 & 3.8907 & 0.55685 & 0.252 & .778 \\
intentions & 4-year college & 111 & 3.8498 & 0.58902 & & \\
& Post graduate & 8 & 3.7500 & 0.46291 & & \\
\hline
\end{tabular}

${ }^{*} p<.05 ;{ }^{* *} p<.01 ;{ }^{* *} p<.001$

Table 7: Visitors' response towards each construct (by occupation)

\begin{tabular}{|c|c|c|c|c|c|c|}
\hline Construct & & $N$ & Mean & S.D. & $F$-value & Sig. \\
\hline \multirow[t]{4}{*}{ Facilities } & Self-employed & 13 & 3.8462 & 0.36300 & 0.712 & .546 \\
\hline & Service industry & 45 & 3.7963 & 0.54575 & & \\
\hline & Governmental & 6 & 3.5000 & 0.39441 & & \\
\hline & Others & 116 & 3.7759 & 0.50229 & & \\
\hline \multirow[t]{4}{*}{ Service quality } & Self-employed & 13 & 3.8154 & 0.33874 & 0.362 & .780 \\
\hline & Service industry & 45 & 3.7667 & 0.34245 & & \\
\hline & Governmental & 6 & 3.9333 & 0.18619 & & \\
\hline & Others & 116 & 3.7966 & 0.40280 & & \\
\hline \multirow[t]{4}{*}{ Experience value } & Self-employed & 13 & 4.1250 & 0.26124 & 0.570 & .982 \\
\hline & Service industry & 45 & 4.1000 & 0.36520 & & \\
\hline & Governmental & 6 & 4.0833 & 0.42753 & & \\
\hline & Others & 116 & 4.0873 & 0.40227 & & \\
\hline \multirow[t]{4}{*}{ Satisfaction } & Self-employed & 13 & 4.2051 & 0.37363 & 1.764 & .156 \\
\hline & Service industry & 45 & 3.8815 & 0.52779 & & \\
\hline & Governmental & 6 & 3.8889 & 0.17213 & & \\
\hline & Others & 116 & 3.9023 & 0.46317 & & \\
\hline \multirow{4}{*}{$\begin{array}{l}\text { Behavioral } \\
\text { intentions }\end{array}$} & Self-employed & 13 & 3.8462 & 0.25975 & 0.062 & .980 \\
\hline & Service industry & 45 & 3.8296 & 0.53476 & & \\
\hline & Governmental & 6 & 3.8889 & 0.17213 & & \\
\hline & Others & 116 & 3.8707 & 0.62442 & & \\
\hline
\end{tabular}

${ }^{*} p<.05 ; \stackrel{* *}{* 0}<.01 ; \stackrel{* * *}{p}<.001$

Table 8: Visitors' response towards each construct (by accompany number, including self) 
International Journal of Social Science and Economic Research

ISSN: $2455-8834$

Volume: 05, Issue: 05 "May 2020"

\begin{tabular}{llccccc}
\hline Construct & & $N$ & Mean & S.D. & F-value & Sig. \\
\hline Facilities & 3 persons or less & 80 & 3.7500 & 0.55245 & 2.391 & .094 \\
& 4-6 persons & 82 & 3.8455 & 0.47052 & & \\
Service quality & 7 persons or more & 18 & 3.5741 & 0.32450 & & \\
& 3 persons or less & 80 & 3.7763 & 0.37829 & 0.473 & .624 \\
& 4-6 persons & 82 & 3.7963 & 0.40596 & & \\
Experience value & 7 persons or more & 18 & 3.8722 & 0.20809 & & \\
& 3 persons or less & 80 & 4.0953 & 0.38783 & 2.084 & .128 \\
& 4-6 persons & 82 & 4.1235 & 0.31334 & & \\
Satisfaction & 7 persons or more & 18 & 3.9444 & 0.13020 & & \\
& 3 persons or less & 80 & 3.8667 & 0.50204 & 1.267 & .284 \\
& 4-6 persons & 82 & 3.9797 & 0.42341 & & \\
Behavioral & 7 persons or more & 18 & 3.8704 & 0.53796 & & \\
intentions & 3 persons or less & 80 & 3.7875 & 0.65656 & 2.711 & .069 \\
& 4-6 persons & 82 & 3.9634 & 0.52117 & & \\
\hline
\end{tabular}

${ }^{*} p<.05 ;{ }^{* *} p<.01 ;{ }^{* * *} p<.001$

Table 9: Visitors' response towards each construct (by accompanied child number)

\begin{tabular}{|c|c|c|c|c|c|c|}
\hline Construct & & $N$ & Mean & S.D. & F-value & Sig. \\
\hline Facilities & $\begin{array}{l}\text { None } \\
1 \text { child } \\
2 \text { children } \\
3 \text { children or more }\end{array}$ & $\begin{array}{l}77 \\
43 \\
44 \\
16\end{array}$ & $\begin{array}{l}3.7749 \\
3.6667 \\
4.0530 \\
3.3125\end{array}$ & $\begin{array}{l}0.53309 \\
0.46434 \\
0.39502 \\
0.14751\end{array}$ & 11.407 & $.000^{* * *}$ \\
\hline Service quality & $\begin{array}{l}\text { None } \\
1 \text { child } \\
2 \text { children } \\
3 \text { children or more }\end{array}$ & $\begin{array}{l}77 \\
43 \\
44 \\
16\end{array}$ & $\begin{array}{l}3.7948 \\
3.7163 \\
3.9273 \\
3.6437\end{array}$ & $\begin{array}{l}0.42578 \\
0.31843 \\
0.33506 \\
0.28976\end{array}$ & 3.410 & $.019^{*}$ \\
\hline $\begin{array}{l}\text { Experience } \\
\text { value }\end{array}$ & $\begin{array}{l}\text { None } \\
1 \text { child } \\
2 \text { children } \\
3 \text { children or more }\end{array}$ & $\begin{array}{l}77 \\
43 \\
44 \\
16\end{array}$ & $\begin{array}{l}4.0325 \\
4.0785 \\
4.2330 \\
4.0391\end{array}$ & $\begin{array}{l}0.35783 \\
0.35352 \\
0.20848 \\
0.20268\end{array}$ & 3.628 & $.014^{*}$ \\
\hline Satisfaction & $\begin{array}{l}\text { None } \\
1 \text { child } \\
2 \text { children } \\
3 \text { children or more }\end{array}$ & $\begin{array}{l}77 \\
43 \\
44 \\
16\end{array}$ & $\begin{array}{l}3.8571 \\
3.8992 \\
4.0606 \\
3.8750\end{array}$ & $\begin{array}{l}0.6056 \\
0.31316 \\
0.37543 \\
0.20638\end{array}$ & 1.857 & .139 \\
\hline $\begin{array}{l}\text { Behavioral } \\
\text { intentions }\end{array}$ & $\begin{array}{l}\text { None } \\
1 \text { child } \\
2 \text { children } \\
3 \text { children or more }\end{array}$ & $\begin{array}{l}77 \\
43 \\
44 \\
16\end{array}$ & $\begin{array}{l}3.6840 \\
3.8992 \\
4.1212 \\
3.8750\end{array}$ & $\begin{array}{l}0.72122 \\
0.41490 \\
0.36720 \\
0.16667\end{array}$ & 6.044 & $.001^{* *}$ \\
\hline
\end{tabular}

${ }^{*} p<.05 ;{ }^{* *} p<.01 ;{ }^{* * *} p<.001$

Table 10: Post-hoc test by LSD and Scheffe for accompanied child number 
International Journal of Social Science and Economic Research

ISSN: $2455-8834$

Volume: 05, Issue: 05 "May 2020"

\begin{tabular}{|c|c|c|c|c|}
\hline Construct & (I) $>$ (J) & Difference & Sig. & Method \\
\hline \multirow[t]{5}{*}{ Facilities } & $(A)>(D)$ & 0.46239 & $\begin{array}{l}.000^{\circ+2} \\
.005^{\circ *}\end{array}$ & $\begin{array}{l}\text { LSD } \\
\text { Scheffe }\end{array}$ \\
\hline & $(\mathrm{B})>(\mathrm{D})$ & 0.35417 & $\begin{array}{l}.010^{* *} \\
.081\end{array}$ & $\begin{array}{l}\text { LSD } \\
\text { Scheffe }\end{array}$ \\
\hline & $(\mathrm{C})>(\mathrm{A})$ & 0.27814 & $\begin{array}{l}.002^{* *} \\
.020^{*}\end{array}$ & $\begin{array}{l}\text { LSD } \\
\text { Scheffe }\end{array}$ \\
\hline & $(\mathrm{C})>(\mathrm{B})$ & 0.38636 & $\begin{array}{l}.000^{* * *} \\
.002^{* *}\end{array}$ & $\begin{array}{l}\text { LSD } \\
\text { Scheffe }\end{array}$ \\
\hline & $(C)>(D)$ & 0.74053 & $\begin{array}{l}.000^{* * *} \\
.000^{* * *}\end{array}$ & $\begin{array}{l}\text { LSD } \\
\text { Scheffe }\end{array}$ \\
\hline \multirow[t]{2}{*}{ Service quality } & $(\mathrm{C})>(\mathrm{D})$ & 0.28352 & $\begin{array}{l}.009^{* *} \\
.080\end{array}$ & $\begin{array}{l}\text { LSD } \\
\text { Scheffe }\end{array}$ \\
\hline & $(\mathrm{C})>(\mathrm{A})$ & 0.20049 & $\begin{array}{l}.002^{* *} \\
.019^{*}\end{array}$ & $\begin{array}{l}\text { LSD } \\
\text { Scheffe }\end{array}$ \\
\hline \multirow[t]{3}{*}{ Experience value } & $(C)>(B)$ & 0.15447 & $\begin{array}{l}.031^{*} \\
.199\end{array}$ & $\begin{array}{l}\text { LSD } \\
\text { Scheffe }\end{array}$ \\
\hline & $(C)>(D)$ & 0.19389 & $\begin{array}{l}.047^{*} \\
.265\end{array}$ & $\begin{array}{l}\text { LSD } \\
\text { Scheffe }\end{array}$ \\
\hline & $(\mathrm{B})>(\mathrm{A})$ & 0.19389 & $\begin{array}{l}.041^{\circ} \\
.240\end{array}$ & $\begin{array}{l}\text { LSD } \\
\text { Scheffe }\end{array}$ \\
\hline \multirow[t]{2}{*}{ Behavioral intentions } & $(\mathrm{C})>(\mathrm{A})$ & 0.43723 & $\begin{array}{l}.000^{* * * *} \\
.001^{\circ *}\end{array}$ & $\begin{array}{l}\text { LSD } \\
\text { Scheffe }\end{array}$ \\
\hline & $(\mathrm{C})>(\mathrm{B})$ & 0.43723 & $\begin{array}{l}.061^{*} \\
.316\end{array}$ & $\begin{array}{l}\text { LSD } \\
\text { Scheffe }\end{array}$ \\
\hline
\end{tabular}

(A) $=$ none; $(\mathrm{B})=1$ child; $(\mathrm{C})=2$ children; $(\mathrm{D})=3$ children or more

${ }^{*} p<.05 ;{ }^{*} p<.01 ;{ }^{* * *} p<.001$

Table 11: Visitors' response towards each construct (by visitation number within a year) 
International Journal of Social Science and Economic Research

ISSN: $2455-8834$

Volume: 05, Issue: 05 "May 2020"

\begin{tabular}{llrcccc}
\hline Construct & & $N$ & Mean & S.D. & F-value & Sig. \\
\hline Facilities & 1 time & 115 & 3.7942 & 0.50645 & 2.866 & .060 \\
& 2 times & 43 & 3.8450 & 0.54116 & & \\
& 3 times or more & 22 & 3.5455 & 0.31363 & & \\
Service quality & 1 time & 115 & 3.8374 & 0.34779 & 2.415 & .092 \\
& 2 times & 43 & 3.7488 & 0.48175 & & \\
Experience value & 3 times or more & 22 & 3.6636 & 0.24985 & & \\
& 1 time & 115 & 4.0837 & 0.36074 & 0.128 & .880 \\
& 2 times & 43 & 4.1134 & 0.31554 & & \\
Satisfaction & 3 times or more & 22 & 4.1023 & 0.27178 & & \\
& 1 time & 115 & 3.8638 & 0.49735 & 2.618 & .076 \\
& 2 times & 43 & 4.0543 & 0.48757 & & \\
Behavioral & 3 times or more & 22 & 3.9394 & 0.16703 & & \\
intentions & 1 time & 115 & 3.8029 & 0.56523 & 1.579 & .209 \\
& 2 times & 43 & 3.9690 & 0.70077 & & \\
\hline$p<.05 ;{ }^{*} p<.01 ;{ }^{* * *} p<.001$ & 22 & 3.9394 & 0.13159 & & \\
& 3 times or more & & & & &
\end{tabular}

As shown in Figure 1, multiple regression analysis showed that customers' "behavioral intentions" was driven mostly by "customer satisfaction" (at $.580^{* * *}$ ), followed by "facilities" (at $.437^{* * *}$ ), "experience value" (at $.384^{* * *}$ ), and "service quality (at $.317^{* * *}$ ). Customers" "satisfaction" was influenced mostly by customers" "experience value" (at $.497^{* * *}$ ), followed by "facilities" (at $.455^{* * *}$ ) and "service quality" (at $.381^{* * *}$ ). Customers' "experience value" was influenced more by "facilities" (at $.476^{* * *}$ ) than "service quality" (at $.408^{* * *}$ ). The regression analysis showed that theme park's facilities would influence visitors' experience value, satisfaction, and behavioral intentions much more than the influence from service quality. Unlike other business entities (such as restaurants, retail stores, or hotels), people pay visits to theme parks for facilities much more than service quality. 


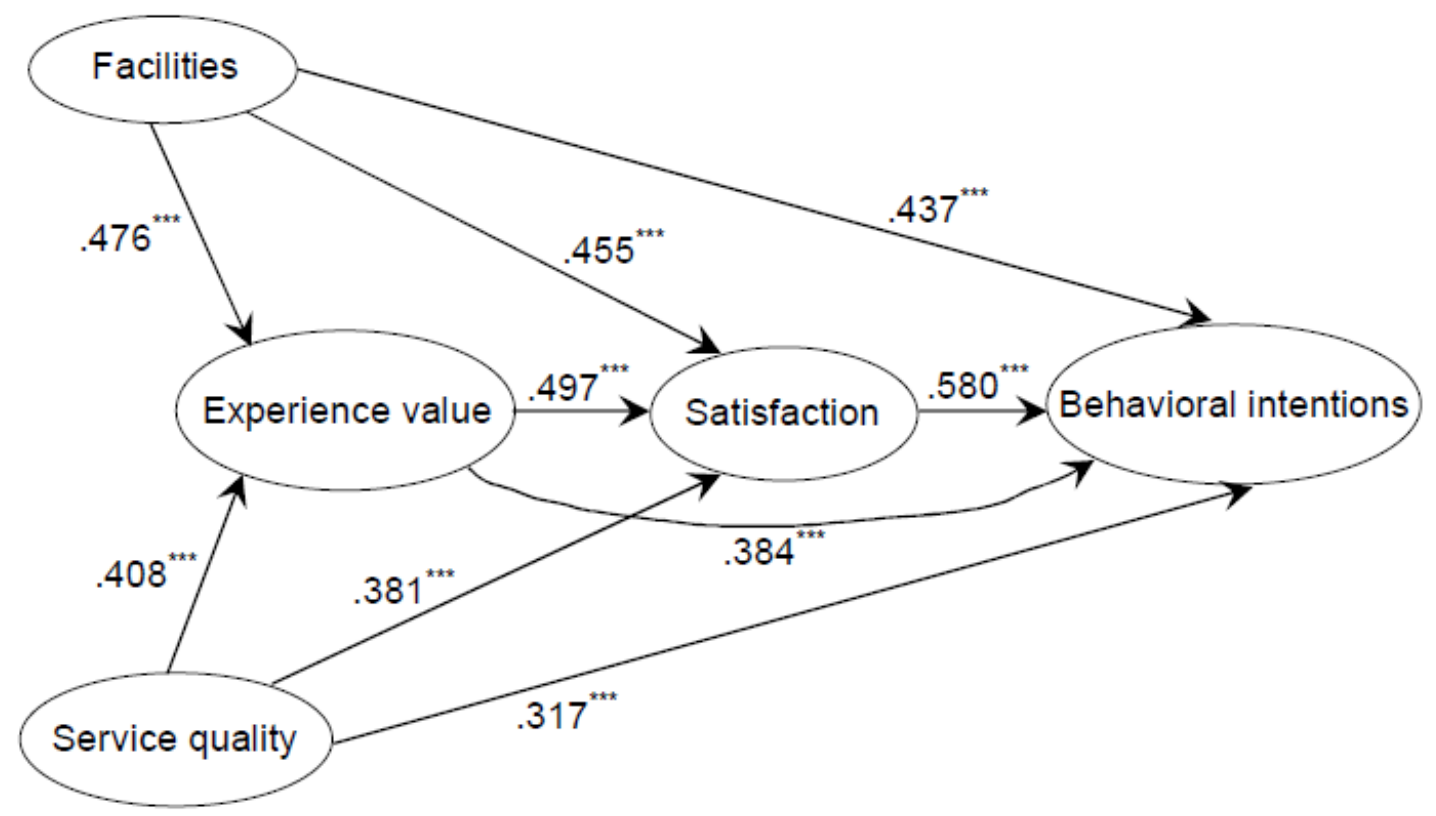

Figure 1: Relationships of facilities, service quality, experience value, satisfaction, and behavioral intentions from urban theme park visitors

\section{CONCLUSION}

The relationships among theme park's facilities, service quality, experience value, customer satisfaction, and behavioral intention were identified that facilities would influence customers' experience value, satisfaction, and behavioral intentions much more than service quality would. The level of influence towards customers' behavioral intentions would go by the order of customer satisfaction, theme park facilities, customers' experience value, and service quality at the very least. Visitors' gender, marital status, education, occupation, total (adults/children) accompanied number, visitation frequency played no statistical significance toward theme park's facilities, service quality, customers' experience value, satisfaction, and behavioral intentions. However, it was found that visitors aged between 26 and 35 years-old are more dissatisfied with the theme park's "facilities" than those of other age groups due to the fact that this particular group of people may be more financially challenged to be pickier toward what they have paid for. Conversely, visitors aged between 36 and 45 years-old are much more likely to pay a return visit due to more financial resources, as well as more likely to have children of suitable age for park visitation.

Visitors who brought two children to the park have much higher satisfaction towards theme park facilities, service quality, and experience value, as well behavioral intentions than those with 


\section{International Journal of Social Science and Economic Research}

ISSN: $2455-8834$

Volume: 05, Issue: 05 "May 2020"

other number of accompanied children. It is reasoned that the age gap between accompanied children may influence the level of enjoyment where visitors with three children enjoyed the park much less. Hence, it is recommended that theme park management shall design activities and/or programs suiting children of all age for simultaneous participation.

\section{REFERENCES}

Buckley, R. (2000). Neat trends: Current issues in nature, eco- and adventure tourism. International Journal of Tourism Research, 2, 437-444.

Bitner, M. J. (1992). Servicescapes: The impact of physical surroundings on customers and employees. Journal of Marketing, 56(2), 57-71.

Chang, C.H., Shu, S., \& King, B. (2014). Novelty in theme park physical surroundings: an application of the stimulus-organism-response paradigm. Asia Pacific Journal of Tourism Research, 19(6), 680-699.

Cheng, Q., Fang, L., \& Chen, H. (2016). Visitors' brand loyalty to a historical and cultural theme park: a case study of Hangzhou Songcheng, China. Current Issues in Tourism, 19(9), 861-868.

de Fontenelle, M. S., \& Zinkhan, G. M. (1993). Gender differences in the perception of leisure: A conceptual model. Advances in Consumer Research, 20(1), 534-540.

Dzeng, R.J., \& Lee, H.Y. (2007). Activity and value orientated decision support for the development planning of a theme park. Expert Systems with Applications, 33, 923-935.

Haahti, A., \& Yavas, U. (2004). A multi-attribute approach to understanding image of a theme park: the case of SantaPark in Lapland. European Business Review, 16(4), 390-397.

Hightower, R., Brady, M. K., \& Baker, T. L. (2002). Investigating the role of the physical environment in hedonic service consumption: An exploratory study of sporting events. Journal of Business Research, 55(9), 697-707.

Hyun, K.H., Min, A., Kim, S.J., \& Lee, J.H. (2016). Investigating cultural uniqueness in theme parks through finding relationships between visual integration of visitor traffics and capacity of service facilities. International Journal of Architectural Computing, 14(3), 247-254. 
Kim, S.S., Lee, C.K., \& Klenosky, D.B. (2003). The influence of push and pull factors at Korean national parks. Tourism Management, 24, 169-180.

Mayer, K. (2002). Human resource practices and service quality in theme parks. International Journal of Contemporary Hospitality Management, 14(4), 169-175.

McClung, G. W. (1991). Theme park selection: Factors influencing attendance. Tourism Management, 12(2), 132-140.

Moutinho, L. (1988). Amusement park visitor behaviour - Scottish attitudes. Tourism Management, 9(4), 291-300.

Millman, A. (2001). The future of the theme park and attraction industry: a management perspective. Journal of Travel Research, 40, 139-147.

Millman, A. (2009). Evaluating the guest experience at theme parks: an empirical investigation of key attributes. International Journal of Tourism Research, 11(4), 373-387.

Samuels, J.B. (1996). Trends in growth and segmentation of the theme/amusement park industry. Visions in Leisure and Business, 15(3), 6-12.

Trischler, J., \& Zehrer, A. (2012). Service design: suggesting a qualitative multistep approach for analyzing and examining theme park experience. Journal of Vacation Marketing, 18(1), $57-71$.

Turley, L. W., \& Fugate, D. L. (1992). The multidimensional nature of service facilities: Viewpoints and recommendations. Journal of Services Marketing, 6(3), 37-45

Wu, H.C., Li, M.Y., \& Li, T. (2018). A study of experiential quality, experiential value, experiential satisfaction, theme park image, and revisit intention. Journal of Hospitality \& Tourism Research, 42(1), 26-73.

Zhang, Y., Li, X.R., \& Su, Q. (2017a). Does spatial layout matter to theme park tourism carrying capacity? Tourism Management, 61, 82-95.

Zhang, Y., Li, X.R., Su, Q., \& Hu, X. (2017b). Exploring a theme parks' tourism carrying capacity: a demand-side analysis. Tourism Management, 59, 564-578. 